\title{
Validação psicométrica do Cocaine Craving Questionnaire-Brief - Versão Brasileira Adaptada para o Crack para dependentes hospitalizados
}

\author{
Psychometric validation of Cocaine Craving Questionnaire-Brief \\ - Brazilian Crack Adapted Version inpatients dependents
}

Renata Brasil Araujoํ, Maria da Graça Tanori de Castro², Rosemeri Siqueira Pedroso30, Paola Lucena dos Santos ${ }^{4}$, Letícia Leite ${ }^{4}$, Marcelo Rossoni da Rocha ${ }^{4}$, Ana Cecília Petta Roselli Marques ${ }^{5}$

\section{RESUMO}

Introdução: O craving (ou fissura) é um fator muito importante no tratamento da dependência de substâncias psicotrópicas. Objetivo: Validar o Cocaine Craving Questionnaire-Brief - Versão Brasileira Adaptada para o Crack. Método: O delineamento foi experimental e seus participantes foram randomizados, em grupos: experimental, para o qual foi apresentada uma imagem de um indivíduo consumindo crack (G1), e controle (G2), para o qual não foi apresentada nenhuma imagem. A amostra foi composta por 109 sujeitos (G1 = 50 e G2 = 59) do sexo masculino, internados por causa da dependência do crack. Os instrumentos utilizados foram: Entrevista Clínica com dados sociodemográficos, CCQ-B Versão Adaptada para o Crack, Escala Analógico-Visual do Craving, Inventários Beck de Ansiedade e de Depressão e o Estímulo Visual indutor de craving para o G1. Resultados: Na análise fatorial, foram encontrados dois fatores: o fator 1, relacionado ao craving propriamente dito, e o fator 2, relacionado à percepção da falta de controle do uso do crack. Os dois fatores apresentaram variância total de $68,84 \%$, e a correlação entre os fatores foi significativa e de baixa intensidade $(r=0,204 ; p=0,041)$. $O$ alfa de Cronbach do seu total de pontos foi 0,85 . $O$ instrumento no total de pontos foi correlacionado com a Escala Analógico-Visual ( $r=$ 0,515; $p<0,01$ ). Conclusão: O CCQ-B - Versão Brasileira Adaptada para o Crack demonstrou ser, psicometricamente, satisfatório para utilização em pesquisas e em ambiente clínico.

\section{ABSTRACT}

Background: The craving is a very important factor in the treatment of drug addiction. Objective: Validate the Cocaine Craving Questionnaire-Brief - Brazilian Crack Adapted Version. Method: Subjects enrolled in this experimental study were randomized into groups: experimental for this group was shown an image of a subject using crack (G1) and control (G2) for this group no pictures were shown. The sample was composed of 109 subjects (G1 = 50 and G2 =59), ma-

1 Pontifícia Universidade Católica do Rio Grande do Sul (PUCRS), Hospital Psiquiátrico São Pedro, Cognitá - Clínica de Terapia Cognitivo-Comportamental.

2 PUCRS.

Recebido em

28/7/2011

Aprovado em

3 Universidade Federal do Rio Grande do Sul (UFRGS), Centro de Pesquisa em Álcool e Drogas.

4 Pontifícia Universidade Católica do Rio Grande do Sul (PUCRS).

5 Universidade Federal de São Paulo (Unifesp). 


\section{Keywords}

CCQ-B, craving, validation, crack/cocaine. les, crack/cocaine dependent inpatients. The assessment instruments were: Clinical Interview with social and demographic data, CCQ-B - Crack Adapted Version; Visual Analogic Scale for Craving, Anxiety and Depression Beck and Inventories Visual Cue to elicit craving. Results: On the factorial analysis two factors were found: Factor 1, related to craving itself, and Factor 2, the perceived lack of control of crack use. The two-factor factorial analysis presented a total variation of $68.84 \%$, and the correlation between these factors was significant and of low intensity $(r=0.204 ; p=0.041)$. A Cronbach's alpha value from total of points of scale was 0.85 . We observed a correlation between the scale total score and the Visual Analogic Scale ( $r=0.515 ; p<0.01)$. Conclusion: The Cocaine Craving Questionnaire-Brief - Brazilian Crack Adapted Version proved to be an adequate psychometric instrument for use in research and in clinical settings.

\section{INTRODUÇÃO}

A cocaína, utilizada na forma de pedra, denominada "crack", é uma substância psicoativa e psicotrópica, cujo consumo tem aumentado, assim como a busca por tratamento'. Esse consumo está associado à alta mortalidade entre os usuários².

O craving ou fissura, por outro lado, é uma variável muito importante na compreensão da dependência, pois influencia a evolução e a gravidade da síndrome de abstinência, como também pode determinar a recaída ${ }^{3,4}$. O craving é um dos critérios da Classificação Internacional de Doenças (CID-10) a ser avaliado no diagnóstico de dependência de substância, sendo descrito no critério A como um desejo forte ou senso de compulsão por consumir a substância ${ }^{5}$; o DSM-IV também lista o craving como um dos critérios a ser considerado, descrito como um desejo persistente. Sabe-se que o craving do crack é muito intenso, fenômeno que pode estar relacionado à via de administração, que produz rápida sensibilização das áreas dopaminérgicas, e da mesma forma ocorre rápida diminuição do seu efeito ${ }^{7}$. Apesar da relevância do craving, não existem muitos instrumentos de avaliação desse sintoma em usuários de crack/cocaína. O craving tem sido pesquisado por alguns autores utilizando "gatilhos" como imagens associadas à substância, para sua indução e aferição, como o estudo conduzido por Tiffany e Drobes ${ }^{8}$ e Zeni e Araujo9. Em nosso meio, Zeni e Araujo utilizaram estímulo visual com fotografias para induzir o craving por nicotina e crack 9 .

Assim, o Cocaine Craving Questionnaire-Brief (CCQ-B) foi desenvolvido por Sussner et al. em $2006^{10}$, a partir do Cocaine Craving Questionnaire-Now (CCQ-Now) ${ }^{11}$, versão longa do instrumento, a qual foi validada no Brasil por Silveira et al. ${ }^{12}$.

Na validação psicométrica da versão original do CCQ-B, foi realizado um estudo de validade convergente com o CCQ-Now, o qual obteve correlação positiva de intensidade muito alta, demonstrando a excelente consistência interna do instrumento ${ }^{10}$. Palihal et al. ${ }^{11}$ também utilizaram o CCQ-B e verificaram que escores mais altos nesse instrumento eram indicadores de recaída em dependentes de cocaína.

Em 2009, foi publicada, no Brasil, a Validação Semântica do CCQ-B e de sua versão adaptada para o crack². A valida- ção semântica é um primeiro passo no processo de validação de um instrumento, já que proporciona a confiança de que as adaptações de linguagem relacionadas a diferentes países foram realizadas, no entanto é necessário que haja uma confirmação de que as propriedades psicométricas do instrumento também estão adequadas, sendo definida a sua forma de análise e seus pontos de corte ${ }^{13}$. Assim, o objetivo deste estudo é realizar, de forma inédita, a validação psicométrica do CCQ-B - Versão Brasileira Adaptada para o Crack em dependentes internados para desintoxicação.

\section{MÉTODO}

\section{Delineamento}

Foi realizado um estudo experimental, no qual a variável craving foi manipulada pela exposição de um estímulo visual. Os pacientes foram divididos, por sorteio, em dois grupos: o G1 (grupo experimental), para o qual foi apresentada uma fotografia de um indivíduo utilizando crack, e G2 (grupo controle), para o qual não foi apresentado nenhum estímulo.

\section{Amostra}

O tamanho da amostra foi definido segundo o critério de Hair Jr., que preconiza um número de 10 sujeitos por variável para a utilização da análise fatorial ${ }^{14}$. A amostra foi composta por 109 indivíduos dependentes de crack/cocaína, segundo os critérios da Classificação Internacional de Doenças (CID-10)5, hospitalizados para desintoxicação em uma unidade masculina especializada, na faixa etária de 18 a 60 anos, com escolaridade mínima de quatro anos. O crack era a droga de preferência, sendo o último uso relatado no mínimo há sete e, no máximo, 21 dias.

Foram excluídos participantes com dificuldades para a leitura e a compreensão do questionário, aferidas na entrevista clínica, que prejudicassem o entendimento das escalas. Os participantes foram divididos, aleatoriamente por sorteio, em dois grupos: no Experimental (G1), foi apresentada uma foto com a imagem de alguém consumindo crack antes da aplicação dos instrumentos, e no Controle (G2), não foi apresentada a foto antes da aplicação. 


\section{Instrumentos}

1) Entrevista composta por informações sociodemográficas e descrição do padrão de uso de substâncias psicoativas e psicotrópicas.

2) Cocaine Craving Questionnaire-Brief (CCQ-B) ${ }^{10}$ : escala com 10 itens, tipo Likert, de 7 pontos, que vai de "discordo totalmente" até "concordo totalmente". O escore do CCQ-B, na validação original ${ }^{10}$, foi obtido a partir da soma de pontos de todas as questões, não sendo subdividida em fatores. Foi utilizada, neste estudo, a Versão Brasileira Adaptada para o Crack, publicada por Araujo et al.13. A versão brasileira, na validação psicométrica, distribuiu-se em dois fatores: o fator 1 representa o constructo do craving, e o fator 2 , a falta de controle do uso do crack. A escala pode ser avaliada a partir de seu escore total (com as questões 4 e 7 invertidas, devendo ser somadas às demais), a partir dos pontos do fator 1 (soma de todas as questões, exceto a 4 e a 7) e do fator 2 (soma das questões 4 e 7 invertidas). Os pontos de corte da escala podem ser observados na tabela abaixo:

Pontos de Corte do CCQ-B - Versão Brasileira Adaptada para o Crack

\begin{tabular}{|c|c|c|c|}
\hline Escala Grau & $\begin{array}{c}\text { CCQ-B - Versão Brasileira } \\
\text { Adaptada para o Crack- } \\
\text { Escore Total }\end{array}$ & Fator 1 & Fator 2 \\
\hline Mínimo & 0 a 11 pontos & 0 a 7 pontos & 0 a 2 pontos \\
\hline Leve & 12 a 16 pontos & 8 a 9 pontos & 3 a 4 pontos \\
\hline Moderado & 17 a 22 pontos & 10 a 11 pontos & 5 a 6 pontos \\
\hline Grave & 23 ou mais pontos & 12 ou mais pontos & 7 ou mais pontos \\
\hline
\end{tabular}

3) Escala Analógico-Visual de avaliação do craving pelo crack no momento (EAV): consiste em uma linha de 10 centímetros, numerados de 0 a 10, que visa medir a intensidade de craving (fissura) pelo crack, significando o 0 "nenhuma fissura" e o 10 "muita fissura". Essa escala já foi utilizada no estudo de Zeni e Araujo, 15

4) Inventário Beck de Ansiedade (BAI), desenvolvido por Steer e Beck ${ }^{16}$, validado para o Brasil por Cunha ${ }^{17}$. Consiste em um questionário que tem por objetivo medir a gravidade dos sintomas de ansiedade e é composto por 21 itens. O escore total é obtido pelo somatório dos escores de cada item. Os pontos de corte para pacientes psiquiátricos são: de 0 a 10: mínimo; de 11 a 19: leve; de 20 a 30: moderado; de 31 a 63: grave.

5) O Inventário Beck de Depressão (BDI) é um dos instrumentos mais usados, tanto na clínica quanto em pesquisa, para avaliar sintomas depressivos. É uma escala de autorrelato, composta por 21 itens, desenvolvida por Steer e Beck ${ }^{16}$ (1993) e validada no Brasil ${ }^{17}$. Os pontos de corte para pacientes psiquiátricos são: 0-11: sintomas mínimos/ausentes; 12-19: sintomas leves; 20-35: sintomas moderados; 36-63: sintomas graves.

6) Estímulo evocador do craving: composto por uma fotografia do tamanho A4 com um indivíduo fazendo uso do crack. Esses estímulos já tinham sido utilizados em outros estudos ${ }^{9,15}$.

\section{Aspectos éticos}

Este estudo foi aprovado pelo Comitê de Ética em Pesquisa do Hospital Psiquiátrico São Pedro. Os dados foram coletados após o indivíduo ter aceitado participar por meio da assinatura do Termo de Consentimento Livre e Esclarecido.

\section{Coleta de dados}

Foi realizada uma entrevista individual em uma sala pequena, com poucos estímulos visuais e sonoros, para os dois grupos: para o Grupo Experimental (G1) foi apresentado, por 60 segundos, o estímulo evocador de craving e para o Grupo Controle (G2) não foi apresentado nenhum estímulo. Para o G1, 1 minuto após a apresentação do estímulo, era feito o preenchimento dos questionários na seguinte ordem: Escala Analógico-Visual, CCQ-B - Versão Brasileira Adaptada para o Crack, BAl e BDI. Para o G2, os mesmos instrumentos, na mesma ordem, eram aplicados após 2 minutos de seus participantes terem entrado na sala, para que houvesse equivalência de tempo entre os dois grupos. Se algum participante relatasse ter craving ao final da aplicação, era realizada a técnica comportamental do relaxamento diafragmático ${ }^{15}$.

\section{Análise de dados}

Os dados foram analisados por meio do software Statistical Package for the Social Sciences (SPSS), versão 17.0, com testes descritivos e de frequências para a análise exploratória dos dados. O teste qui-quadrado e o teste exato de Fisher foram utilizados para analisar a associação entre variáveis categóricas; o teste t de Student, para amostras independentes, para comparar G1 e G2 quanto às variáveis contínuas e na validade discriminante; o coeficiente de correlação linear de Pearson foi utilizado na validade convergente e de critério e no estudo correlacional; o alfa de Cronbach, no estudo de confiabilidade; o Kaiser-Meyer-Olkin (KMO), o teste de Bartlett e a ANOVA, na análise fatorial e a regressão linear para verificar o modelo de composição do craving. O nível de significância utilizado foi $5 \%$.

\section{RESULTADOS}

A amostra de 109 dependentes foi distribuída em: G1, com 50 sujeitos (45,87\%), e G2, com 59 sujeitos (54,13\%). Todos, exceto um paciente, iniciaram o uso de cocaína pela via inalada e depois passaram para o uso do crack. O tempo médio de diferença entre o início do uso de uma via de administração para a outra foi de 5,27 anos (SD = 5,01, mínimo $=-3$; máximo $=29$ ). Quanto ao uso de outras substâncias, 96,33\% eram dependentes de maconha, 91,7\%, de nicotina e 18,35\%, de álcool. Não houve diferença significativa entre os grupos com relação às variáveis analisadas (Tabela 1). 
Tabela 1. Comparação entre médias e (desvios-padrão) entre grupo 1 e grupo 2, segundo aspectos sociodemográficos, padrão de consumo de substâncias e momentos do tratamento/abstinência

\begin{tabular}{|c|c|c|c|c|}
\hline Variáveis & $\begin{array}{l}\text { Grupo } 1 \\
(n=50)\end{array}$ & $\begin{array}{l}\text { Grupo } 2 \\
(\mathrm{n}=59)\end{array}$ & $\mathrm{T}$ & $\mathbf{P}$ \\
\hline Idade & $29,09( \pm 6,34)$ & $26,95( \pm 6,81)$ & 1,648 & 0,102 \\
\hline Anos de estudo & $7,70( \pm 2,24)$ & $8,53( \pm 2,73)$ & $-1,663$ & 0,100 \\
\hline Tempo de internação & $11,67( \pm 9,31)$ & $14,15( \pm 5,22)$ & $-1,545$ & 0,111 \\
\hline Idade do início do uso da cocaína & $17,14( \pm 2,44)$ & $17,61( \pm 4,03)$ & $-0,666$ & 0,507 \\
\hline Tempo do último uso de cocaína (dias) & $436,46( \pm 778,34)$ & $254,51( \pm 341,25)$ & 1,523 & 0,132 \\
\hline Quantidade de cocaína utilizada/semana (gramas) & $14,51( \pm 21,58)$ & $8,01( \pm 13,99)$ & 1,648 & 0,103 \\
\hline Idade do início do uso de crack & $23,30( \pm 6,11)$ & $22,38( \pm 6,42)$ & 0,739 & 0,461 \\
\hline Tempo do último uso de crack (dias) & $12,10( \pm 10,01)$ & $15,31( \pm 10,79)$ & $-1,732$ & 0,080 \\
\hline Quantidade de crack utilizada/ semana (gramas) & $14,68( \pm 16,57)$ & $11,27( \pm 14,32)$ & $-1,534$ & 0,122 \\
\hline Ansiedade (Inventário Beck de Ansiedade) & $16,11( \pm 38,65)$ & $15,88( \pm 32,81)$ & 0,655 & 0,333 \\
\hline Depressão (Inventário Beck de Depressão) & $14,07( \pm 9,22)$ & $15,97( \pm 8,76)$ & 0,822 & 0,524 \\
\hline
\end{tabular}

\section{Confiabilidade}

Para avaliar a consistência interna do instrumento, foram calculados os valores do alfa de Cronbach no questionário como um todo e de seus dois fatores. $\mathrm{O}$ alfa total foi de 0,85 (10 itens), o do fator 1 foi 0,93 (8 itens) e o do fator 2 foi 0,66 ( 2 itens). O método split-half foi utilizado para avaliar a confiabilidade do instrumento, sendo obtido o coeficiente 0,88 .

\section{Análise fatorial}

O Kaiser-Meyer-Olkin (KMO) e o teste de Bartlett foram utilizados com o intuito de comprovar a adequação dos dados do CCQ-B para a análise fatorial. Os resultados deles foram, respectivamente, 0,851 e $p<0,001$ e comprovaram que a utilização da ANOVA seria adequada para a validação da escala. Na tabela 2, estão distribuídas as questões nos fatores da escala a partir da utilização da rotação promax.

O critério utilizado para a colocação dos itens nos fatores foi o descrito por Tiffany e Drobes ${ }^{18}$, os quais consideraram, na validação do Questionnaire of Smoking Urge (QSU), como pertencentes a um determinado fator itens com carga fatorial igual ou superior a 0,40, cuja carga no outro fator fosse menor do que 0,25 , devendo a diferença entre ambas ser de, no mínimo, 0,20.

Os fatores 1 e 2 do CCQ-B apresentaram autovalor de 5,50 e 1,39 e variância de 54,98\% e 13,86\%, respectivamente, sendo o total da variância equivalente a 68,84\% e a correlação entre os dois fatores significativa e de baixa intensidade $(r=0,204 ; p=0,041)$.

\section{Validade convergente e de critério}

A validade convergente e de critério do CCQ-B foi mensurada, respectivamente, por meio da análise das correlações do CCQ-B e seus fatores com os escores da Escala Analógico-Visual e pelas correlações do CCQ-B entre seus fatores (Tabela 3).
Tabela 2. Questões do CCQ-B - Versão Brasileira Adaptada para o Crack e distribuição fatorial

\begin{tabular}{lcc}
\hline & \multicolumn{2}{c}{ Amostra $=109$} \\
\cline { 2 - 3 } Questões & Fator 1 & Fator 2 \\
\cline { 2 - 3 } CCQ-B 1 & $0,734^{*}$ & 0,234 \\
CCQ-B 2 & $0,795^{*}$ & 0,049 \\
CCQ-B 3 & $0,935^{*}$ & $-0,035$ \\
CCQ-B 4 & $-0,033$ & $0,830^{*}$ \\
CCQ-B 5 & $0,819^{*}$ & 0,016 \\
CCQ-B 6 & $0,739^{*}$ & $-0,098$ \\
CCQ-B 7 & $-0,030$ & $0,836^{*}$ \\
CCQ-B 8 & $0,774^{*}$ & $-0,104$ \\
CCQ-B 9 & $0,912^{*}$ & $-0,075$ \\
CCQ-B 10 & $0,847^{*}$ & 0,031 \\
\hline
\end{tabular}

* Participa da composição do fator.

Tabela 3. Correlações do CCQ-B - Versão Brasileira Adaptada para o Crack, fatores 1 e 2 e a Escala Analógico-Visual

\begin{tabular}{lcccc}
\hline Amostra $=109$ & CCQB Total & Fator 1 & Fator 2 & $\begin{array}{c}\text { Escala } \\
\text { Analógico- } \\
\text { Visual }\end{array}$ \\
\hline CCQ-B total & 1,00 & $0,925^{* *}$ & $0,550^{* *}$ & $0,515^{* *}$ \\
Fator 1 & $0,925^{* *}$ & 1,00 & $0,209^{*}$ & $0,447^{* *}$ \\
Fator 2 & $0,550^{* *}$ & $0,209^{*}$ & 1,00 & $0,350^{* *}$ \\
EAV & $0,515^{* *}$ & $0,447^{* *}$ & $0,350^{* *}$ & 1,00 \\
\hline
\end{tabular}

${ }^{*} p<0,05 ; *{ }^{* *} p<0,01$

\section{Validade discriminante}

Foi avaliada pelo escore total do CCQ-B, pelo escore de seus fatores e pela pontuação na Escala Analógico-Visual, no G1 e no G2 (Tabela 4). 
Tabela 4. Comparação de médias e (desvios-padrão) do craving entre grupo 1 e grupo 2

\begin{tabular}{lcccc}
\hline Variáveis & $\begin{array}{c}\text { Grupo 1 } \\
(\mathbf{n}=50)\end{array}$ & $\begin{array}{c}\text { Grupo } \\
(\mathbf{n}=59)\end{array}$ & $\mathbf{T}$ & $\mathbf{P}$ \\
\hline CCQ-B total & $19,82( \pm 12,27)$ & $15,80( \pm 6,03)$ & 2,045 & 0,044 \\
CCQ-B fator 1 & $13,38( \pm 10,29)$ & $10,22( \pm 3,42)$ & 2,028 & 0,045 \\
CCQ-B fator 2 & $6,44( \pm 4,18)$ & $5,57( \pm 4,23)$ & 1,006 & 0,317 \\
Escala Analógico-Visual & $5,22( \pm 2,14)$ & $3,45( \pm 2,02)$ & 4,617 & 0,001 \\
\hline
\end{tabular}

\section{Modelo do craving}

Por meio da regressão linear, foi verificado quais eram as variáveis indicativas do total de pontos do CCQ-B. O modelo que melhor explicou essa variável foi o composto por: total de pontos do BDI e do BAI $\left[R^{2}=0,949 ; F(4,15)=23,009\right.$; $p<0,001]$. O valor do coeficiente beta do total de pontos do BDI foi de 0,584 ( $p=0,01)$ e o do BAl, de 0,493 ( $p=0,05)$. As variáveis excluídas pela regressão linear foram: idade, escolaridade, estado civil, idade de início do uso de cocaína e de crack, quantidade consumida de cocaína e de crack e último consumo de cocaína e de crack.

\section{Craving e fatores associados}

O coeficiente de correlação linear de Pearson foi empregado no estudo correlacional entre o total de pontos do CCQ-B e algumas variáveis. Foram encontradas correlações positivas de baixa intensidade $(0,20<r<0,40)$ a muito baixa $(0<r<$ $0,20)$ e com os sintomas de depressão pelo BDI $(r=0,382$; $p=0,010)$ e a quantidade de crack consumida em gramas por semana ( $r=0,194 ; p=0,044)$ e correlação negativa de intensidade baixa com o tempo do último uso de tabaco $(r=-0,230 ; p=0,028)$. O fator 1 teve correlação positiva de intensidade baixa apenas com os sintomas de depressão pelo $\mathrm{BDI}(r=0,335 ; \mathrm{p}=0,025)$, e o fator 2 teve correlação positiva de intensidade baixa com os sintomas de depressão, BDI ( $r$ = 0,299; $p=0,046$ ), e correlação negativa de baixa intensidade com o tempo do último uso de tabaco $(r=-0,234 ; p=0,025)$.

Não foi identificada correlação entre o total do CCQ-B, nem entre os dois fatores dessa escala e idade, escolaridade em anos de estudo, idade de início do uso do crack, tempo de internação, sintomas de ansiedade (BAl) e tempos do último uso do crack, da cocaína inalada, da maconha e do álcool ( $p>0,05)$.

\section{DISCUSSÃO}

\section{Propriedades da escala}

O CCQ-B - Versão Brasileira Adaptada para o Crack demonstrou ser um instrumento adequado para mensurar o craving do crack, a partir da avaliação de suas propriedades psicométricas. Pela análise fatorial, foram obtidos dois fatores com cargas fatoriais altas, demonstrando a forte participação de cada questão nos respectivos fatores. Os resultados foram adequados, segundo os parâmetros de Tiffany e Drobes ${ }^{18}$. O fator 1 seria o craving e o fator 2, a falta de controle do uso do crack. Sussner et al. ${ }^{10}$ e Palihal et al. ${ }^{11}$ não realizaram análise fatorial do CCQ-B, no entanto, na análise fatorial da escala original, o CCQ-Now ${ }^{11}$, foi obtida uma solução de quatro fatores, os quais continham questões relativas a duas ou mais das seguintes categorias: desejo de usar cocaína, intenção e planejamento de usar cocaína, antecipação do efeito positivo, antecipação do alívio dos sintomas de abstinência ou da disforia e falta de controle do uso da cocaína. Assim, Tiffany et al.? consideraram que as categorias já destacadas na validação do $\mathrm{QSU}^{18}$ e que comporiam o craving pelo tabaco também fariam parte do craving pela cocaína, no entanto salientaram a presença de uma nova categoria: a falta de controle do uso. A presença dessa nova categoria igualmente foi observada em nosso estudo.

Foram encontradas, na validade de critério, correlações positivas do total de pontos com os dois fatores, no entanto a correlação com o fator 2 foi de intensidade moderada e com o fator 1, muito alta, demonstrando que a avaliação do somatório de pontos da escala e de seu fator 1 mensurou o mesmo constructo. A correlação entre os dois fatores, por outro lado, foi de intensidade baixa ${ }^{19}$. Isso significa que o fator 1, denominado de "craving", mensura o mesmo que o total de pontos da escala, sendo facultativo utilizar este último cálculo, por outro lado, demonstra que o fator 2 - falta de controle do uso do crack - não faz parte do constructo craving, sendo uma análise separada. O fato de o fator 2 aparecer como um fator separado pode estar relacionado ao fato de o seu craving ser muito intenso ${ }^{15}$. Mas deve-se destacar que as questões que compõem o fator 2 são invertidas, o que pode ter influenciado nesse resultado, como já alertado por Tiffany et al.?

O instrumento apresentou um nível satisfatório de consistência interna e de confiabilidade, de acordo com Bisquerra et al. ${ }^{19}$. Apesar de o fator 2 ter um valor de alfa inferior a 0,70 , foi muito próximo dele $(0,66)$ e pode ser justificado pelo pequeno número de questões - apenas duas - que compõem esse fator, não prejudicando a confiabilidade do instrumento. A confiabilidade do CCQ-B já havia sido encontrada em outros estudos ${ }^{10,11}$.

$\mathrm{Na}$ análise convergente, pode-se observar que o CCQ-B em seu total de pontos e seu fator 1 tiveram correlações positivas e moderadas e, em seu fator 2, uma correlação positiva de intensidade baixa com a Escala Analógico-Visual para mensurar o craving, resultados também destacados por Palihal et al. ${ }^{11}$ e Sussner et al. ${ }^{10}$. O fato de as correlações serem de intensidades moderada e baixa, pode-se inferir, ocorreu em virtude de a Escala Analógico-Visual analisar o craving de forma uni e não multidimensional, como é o caso do CCQ-B. Outro ponto que se observa na clínica e que responderia pelos valores dessas correlações é que os dependentes, quando questionados diretamente a respeito do craving como 
desejo, o confundem com a intenção de realizar esse desejo, outro componente do craving ${ }^{4}$. A crítica à utilização de escalas unidimensionais para avaliar o craving já foi feita por alguns autores, os quais destacam o aspecto multifatorial do fenômeno $0^{4,18}$.

Como não houve diferenças significativas entre os grupos experimental e controle em variáveis que poderiam interferir no craving, foi possível realizar a validade descriminante da escala. A apresentação do estímulo visual foi efetiva em induzir o craving, como ocorre em outros estudos ${ }^{8,9,15}$. Segundo a validade descriminante, pode-se verificar que o CCQ-B foi sensível para perceber diferenças entre o craving nos grupos experimental e controle, tanto pelo seu total de pontos quanto pelo seu fator 1 (Craving). O fator 2 (falta de controle do uso do crack), por outro lado, não variou nos dois grupos. Isso faz com que se infira que a falta de controle não faça parte do constructo craving ${ }^{4}$, mas que seja uma consequência dele, o que torna mais consistente a constatação da baixa correlação entre os dois fatores da escala. Neste ponto, os achados são discordantes dos encontrados por Tiffany et al. ${ }^{7}$, que acreditam que a falta de controle não é uma consequência do craving, mas que faz parte do automatismo do comportamento de usar a droga que ocorre em indivíduos com uma longa história de consumo. A falta de controle do uso da substância não havia sido destacada como um componente do craving em estudo relacionado a outra substância utilizada pela via fumada, o tabaco $^{18}$.

O modelo que melhor explicou o total de pontos do CCQ-B foi o composto por sintomas de depressão e de ansiedade, o que está de acordo com o já defendido por alguns autores como o uso da substância como alívio do afeto negativo fazer parte do constructo craving $^{4,7,18}$.

\section{Correlações com sintomas psiquiátricos}

Na análise correlacional, foi observada correlação positiva de intensidade baixa entre o total de pontos do CCQ-B, entre os dois fatores, e os sintomas de depressão no BDI, o que está de acordo os resultados da literatura ${ }^{10,11}$, sendo encontrada correlação positiva, mas de intensidade muito baixa, do total de pontos da escala com a quantidade de crack consumida em gramas por semana. Tanto os pacientes que apresentavam mais sintomas de depressão, quanto aqueles que consumiam mais crack, em nossa amostra, tinham craving mais intenso, no entanto apenas os deprimidos percebiam uma maior falta de controle do uso do crack. Tiffany et al. ${ }^{7}$ encontraram correlações positivas do total de pontos dessa escala e a frequência de utilização da cocaína nos últimos seis meses utilizando o CCQ-Now.

Foram encontradas correlações negativas de intensidade baixa entre o total de pontos do CCQ-B e entre o fator 2 e o tempo do último uso de tabaco, ou seja, quanto menos tempo passou do último uso do tabaco, maior é o craving pelo crack e mais o indivíduo percebe-se como não tendo o controle do uso desta última substância, o que está de acordo com Zeni e Araujo ${ }^{9}$, que salientaram a associação entre o craving por ambas as substâncias e a importância do tratamento concomitante das duas dependências?. Pode-se destacar um dado que contrasta com o de outros estudos ${ }^{7,10}$, que foi não encontrar a correlação entre o craving e sintomas de ansiedade pelo BAI.

Não foram encontradas correlações entre o craving e fatores associados à gravidade da dependência como: idade de início do uso do crack, tempo de internação e tempo decorrido do último uso de crack e de cocaína inalada. Pode-se inferir que esses achados estejam relacionados, no caso do crack, à pouca variação dessas variáveis na amostra pesquisada e, no caso da cocaína inalada, pelo fato de o craving pelo crack ter suas especificidades como seu efeito fugaz e característico ritual de consumo ${ }^{15}$. Deve-se destacar que Sussner et al..$^{10}$ também não encontraram associação entre o craving medido pelo CCQ-B e o número de dias de consumo de cocaína no último mês.

\section{Limitações}

É um estudo com amostra composta apenas por pacientes adultos, do sexo masculino e em regime de internação, o que pode influenciar os pontos de corte do questionário estudado. Outras limitações deste estudo foram a não apresentação de um estímulo neutro para o G2 e a não mensuração do craving nos dois grupos antes da apresentação do estímulo visual.

Diferentes ambientes de tratamento devem ser estudados, pois a maioria dos dependentes é encontrada no tratamento ambulatorial. Mulheres e adolescentes dependentes devem compor novas amostras de pesquisas. Perspectivas futuras apontam para estudos que também utilizem biomarcadores na avaliação do craving.

\section{CONCLUSÃO}

Conclui-se que o CCQ-B - Versão Brasileira Adaptada para o Crack possui propriedades psicométricas satisfatórias, podendo ser um instrumento de avaliação do craving em pesquisas, como também em ambientes clínicos especializados no tratamento da dependência, no entanto ressaltamos que o mesmo deve ser avaliado em outros contextos clínicos. A amostra neste estudo permitiu sua avaliação em pacientes masculinos, adultos e hospitalizados, mas são necessários estudos com outros sujeitos.

\section{AGRADECIMENTOS}

Ao Dr. Bradley Sussner e ao Dr. Stephen Tiffany, pela permissão de validar o CCQ-Brief - Versão Brasileira e o CCQ-Brief 
- Versão Brasileira Adaptada para o Crack. Ao referir-se às versões brasileiras do CCQ-Brief, solicitamos que sejam citados os artigos Tiffany et al. ${ }^{7}$ e Sussner et al. ${ }^{6}$ encontrados nas referências deste artigo.

\section{REFERÊNCIAS}

1. Guimarães CF, Santos DVV, Freitas RC, Araujo RBI. Perfil do usuário de crack e fatores relacionados à criminalidade em unidade de internação para desintoxicação no Hospital Psiquiátrico São Pedro de Porto Alegre (RS). Rev Psiquiatr Rio Gd Sul. 2008;30(2):101-8.

2. Dias AC, Araújo MR, Dunn J, Sesso RC, De Castro V, Laranjeira R. Mortality rate among crack/cocaine-dependent patients: a 12-year prospective cohort study conducted in Brazil. J Subst Abuse Treat. 2011;41(3):273-8.

3. Marques AC, Seibel SD. 0 craving. In: Seibel SD, Toscano Jr. A, editores. Dependência de drogas. São Paulo: Atheneu; 2001. p. 239-48.

4. Araujo RB, Oliveira MS, Pedroso RS, Miguel AC, Castro MGT. Craving e dependência química: conceito, avaliação e tratamento. J Bras Psiquiatr. 2008;57(1):57-63.

5. Organização Mundial de Saúde. Classificação de transtornos mentais e de comportamento da CID-10: descrições clínicas e diretrizes diagnósticas. Porto Alegre: Artes Médicas; 1993.

6. American Psychiatric Association - DSM-IV-TR. Manual Diagnóstico e Estatístico de Transtornos Mentais. 4. ed. Texto revisado. Porto Alegre: Artmed; 2002.

7. Tiffany ST, Singleton E, Haertzen CA, Henningfield JE. The development of a cocaine craving questionnaire. Drug Alcohol Depend. 1993;34:19-28.
8. Tiffany ST, Drobes DJ. Imagery and smoking urges: the manipulation of affective content. Addict Behav. 1990;15(6):531-9.

9. Zeni TC, Araujo RB. Relação entre o craving por tabaco e o craving por crack em pacientes internados para desintoxicação. J Bras Psiquiatr. 2011;60(1):28-33.

10. Sussner B, Smelson DA, Rodrigues S, Kline A, Losonczy M, Ziedonis D. The validity and reliability of a brief measure of cocaine craving. Drug Alcohol Depend. 2006;83(3):233-7.

11. Palihal P, Hyman SM, Sinha R. Craving predicts time to cocaine relapse: further validation of the now and brief versions of the Cocaine Craving Questionnaire. Drug Alcohol Depend. 2008:93(3):252-9.

12. Silveira $D X$, Fernandes $M$, Silveira ED, Jorge MR. Cocaine craving questionnaire: assessing craving among cocaine users in Brazil. Psychiatry Res. 2006;142:257-9.

13. Araujo RB, Pedroso RS, Castro MGT. Adaptação transcultural para o idioma português do Cocaine Craving Questionnaire - Brief. Rev Psiq Clín. 2010;37(5):195-8.

14. Hair Jr JF, Anderson RE, Tatham RL, Black WC. Multivariate Data Analysis. 5. ed. Upper Saddle River: Prentice Hall; 1998.

15. Zeni TC, Araujo RB. 0 relaxamento respiratório no manejo do craving e dos sintomas de ansiedade em dependentes de crack. Rev Psiquiatr Rio Gd Sul. 2009;31(2):116-9.

16. Steer RA, Beck A. Beck Depression Inventory (BDI). In: Sederer LI, Dickey B. Outcomes assessment in clinical practice. Baltimore: Williams \& Wilkins; 1996. p. 100-4.

17. Cunha JA. Manual da versão em português das Escalas Beck. São Paulo: Casa do Psicólogo; 2001. $171 \mathrm{p}$.

18. Tiffany ST, Drobes DJ. The development and initial validation of a questionnaire on smoking urges. Br J Addict. 1991;86:1467-76.

19. Bisquerra R, Sarriera JC, Martinez F. Introdução à estatística - enfoque informático com 0 pacote estatístico SPSS. Porto Alegre: Artmed; 2004. 